\title{
Single-blind control
}

David F Marks

Potential competing interests: The author(s) declared that no potential competing interests exist.

Single-blind control is when the investigator but not the participant is aware of the condition the participant has been allocated to. 